\title{
Polarization-sensitive optical coherence tomography as a tool to visualize the fiber direction of retinal nerves and peripapillary sclera
}

\author{
Joy Willemse ${ }^{1, *}$, Frank Verbraak ${ }^{2}$, and Johannes de Boer ${ }^{1,2}$ \\ ${ }^{1}$ LaserLab, Department of Physics and Astronomy, Vrije Universiteit Amsterdam, de Boelelaan 1081, 1081 HV Amsterdam, the \\ Netherlands \\ ${ }^{2}$ Amsterdam UMC, Vrije Universiteit Amsterdam, Ophthalmology Dept., de Boelelaan 1117, Amsterdam, the Netherlands
}

\begin{abstract}
Polarization sensitive optical coherence tomography (PS-OCT) has been used to visualize the orientation of the nerves in the retinal nerve fiber layer (RNFL) and to visualize the orientation of the collagen fibers in peripapillary sclera in retinas of healthy volunteers. Optic axis orientation images clearly visualize the nerve fibers leaving the optic nerve head $(\mathrm{ONH})$ in all radial directions. Sclera orientation images show that the sclera consist of two layers, an inner layer with an orientation parallel to the RNFL orientation, and a deeper layer where the collagen is circularly oriented.
\end{abstract}

\section{Purpose}

Optical coherence tomography (OCT) is an imaging technique which is used in ophthalmology to create highresolution retinal images. Additional information on the tissue can be obtained with polarization sensitive OCT (PS-OCT), where polarization changing effects of the tissue can be captured and included in the analysis of the backscattered light. In the retina, this includes birefringence of the retinal nerve fiber layer and sclera, and depolarization of the retinal pigment epithelium (RPE). Furthermore, the optic axis orientation can be extracted, which shows the (relative) direction of fibers. It is hypothesized that changes in birefringence of the retinal nerve fiber layer (RNFL) may precede the thinning of the RNFL in the early symptoms of glaucoma [1]. Therefore, the possibilities to image RNFL phase retardation and fiber orientation have been explored previously $[2,3]$. In addition, deviations in peripapillary sclera structure might precede damage to the optic nerve head $(\mathrm{ONH})$ [4]. In this experimental proof-of-concept study polarization-sensitive optical coherence tomography (PS-OCT) was used in healthy volunteers to evaluate the local birefringence and direction of the fibers of the RNFL and sclera.

\section{Methods}

Seven healthy volunteers were scanned with our in-house built PS-OCT system. PS-OCT imaging included intensity, local phase retardation, degree of polarization uniformity (DOPU), and relative optic axis images. The data was corrected for polarization mode dispersion (PMD) by an algorithm by Braaf et al. [5]. Local birefringence images and optic axis orientation were extracted using the differential Mueller matrix method developed by Villiger et al. [6]. This algorithm was modified to correct for the birefringence of preceding layers. To visualize the birefringence with higher signalto-noise ratio, optic axis uniformity $(\mathrm{OAxU})$ images were used [7]. The setup contained a swept-source laser centered at $1060 \mathrm{~nm}$ with a repetition rate of $100 \mathrm{kHz}$. Segmentation on the RNFL was performed by using a combination of DOPU, OAxU and intensity images. Segmentation on the sclera was performed using OAxU images only. Sclera orientation en face images were created by averaging the optic axes in the first $150 \mu \mathrm{m}$ of sclera pixels.

\section{Results}

Figure 1a shows an en face intensity map of the retina of a healthy volunteer The RNFL was segmented automatically, and an en face RNFL thickness map was made (figure 1b). Local birefringence of the RNFL is shown in figure 1c. The relative orientation of the RNFL was found by averaging the measured local optic axis of the RNFL in depth. Figure 1d shows the en face intensity map with an overlay of red lines which delineate the orientation of the RNFL. It can be seen that the nerve fibers are leaving the optic nerve head $(\mathrm{ONH})$ in all radial directions. Minimum RNFL thickness to extract the relative optic axis reliably was about $60 \mu \mathrm{m}$.

\footnotetext{
* Corresponding author: j.willemse@,vu.nl
} 

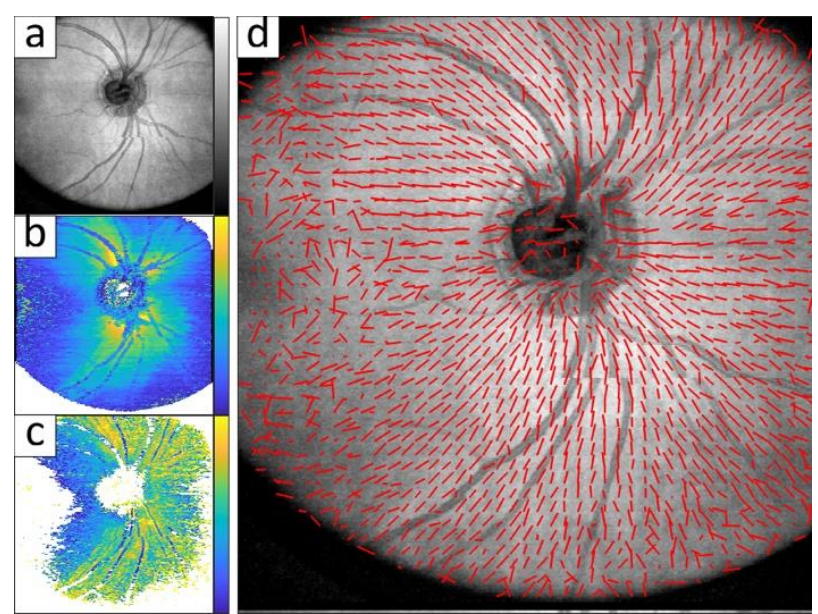

Fig. 1. PSOCT images of a healthy volunteer (a) Intensity en face image, (b) RNFL thickness image, (c) local birefringence image and (d) Intensity en face image overlaid with RNFL orientation as red lines. Color range in (b) indicates $0-300 \mu \mathrm{m}$. Color range in (c) indicates $0.5-0.15 \% / \mu \mathrm{m}$. Images sizes: 5.7 x $6.2 \mathrm{~mm}$ (depth $\mathrm{x}$ height).

Figure 2 shows the orientations measured in the sclera of another healthy volunteer. Figure $2 \mathrm{~b}$ shows the orientations measured in the sclera, where the white dotted lines correspond to the B-scans shown on the right of the image. These B-scans show the intensity with an overlay of the optic axis orientation for pixels with high OAxU. Two different orientations where measured for the sclera. The inner layer of sclera consists of collagen oriented parallel to the RNFL, i.e. radial from the ONH. A deeper layer was found where the orientation is circular around the $\mathrm{ONH}$. Close to the $\mathrm{ONH}$, the second layer displaces the first layer, as indicated with the red arrows shown in figure $2 \mathrm{~d}$. This results in a ring of different orientation as can be seen in the sclera orientation en face image (Figure 2b).

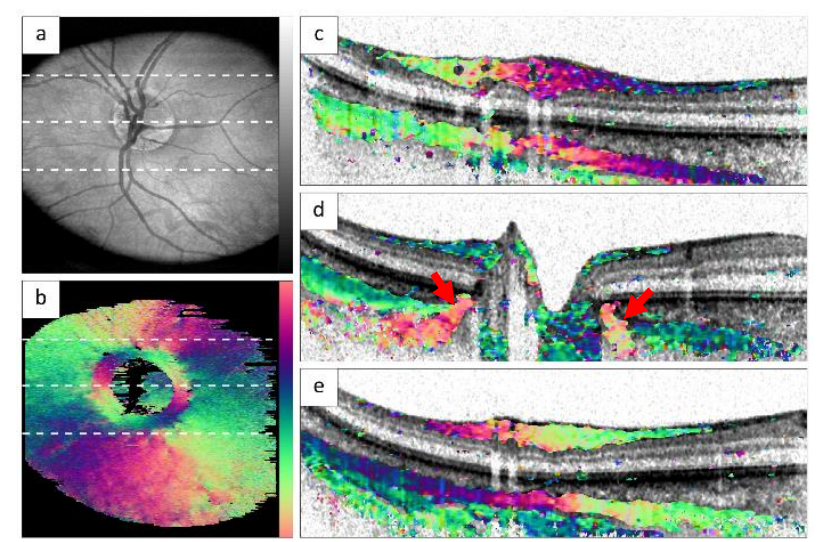

Fig. 2. Sclera orientation images of a healthy volunteer (a) Intensity en face image, (b) sclera orientation en face image, (c-e) cross sections of frames indicated with white in the en face images. Intensity is overlaid with the orientation of pixels with high OAxU. Color range in (b) indicates $0-180^{\circ}$. Images sizes: $(\mathrm{a}-\mathrm{b}) 5.7 \times 6.2 \mathrm{~mm}$ (depth $\mathrm{x}$ height), (c-e) $1.2 \times 6.2 \mathrm{~mm}$ (depth $\mathrm{x}$ height)
Eyes of five out of seven volunteers showed these two orientations for the sclera, with the second layer coming closer to the surface in a ring around the ONH. In two volunteers, the retina absorbed the light before reaching the sclera, leaving too little signal to perform the analysis.

\section{Conclusions}

PS-OCT has been used to successfully extract the birefringence and orientation of the retinal nerves and sclera. To our knowledge, PSOCT is the only technique which is able extract $3 \mathrm{D}$ orientation of fibrous structures of the human retina in vivo. The relative direction of the fibers around the $\mathrm{ONH}$ have been visualized, demonstrating a radial pattern for RNFL and the first layer of sclera. A second sclera layer with circumferential orientation was found. In RNFL, the fiber direction and its changes could possibly be correlated with areas of visual field loss, and might be used to identify areas of (expected) RNFL loss in glaucoma. Deviations in peripapillary sclera structure might precede damage to the optic nerve head $(\mathrm{ONH})$ in glaucoma. In future research, we will scan glaucoma patients with the PS-OCT system to test these hypotheses.

\section{References}

[1] B. Cense, T. C. Chen, B. H. Park, M. C. Pierce, and J. F. de Boer, Invest. Ophth. Vis. Sci. J. 45, 2606-2612 (2004).

[2] S. Zotter, M. Pircher, E. Gotzinger, T. Torzicky, H. Yoshida, F. Hirose, S. Holzer, J. Kroisamer, C. Vass, U. Schmidt-Erfurth, and C. K. Hitzenberger, Invest Ophth Vis Sci 54, 72-84 (2013).

[3] M. Sugita, M. Pircher, S. Zotter, B. Baumann, P. Roberts, T. Makihira \& C. K. Hitzenberger, Biomed Opt Express, 6(3), 1030-1054 (2015).

[4] C. F. Burgoyne, J. C. Downs, A. J. Bellezza, and R. T. Hart, Invest. Ophth. Vis. Sci. J. 45(12), 4388-4399 (2004).

[5] B. Braaf, K. A. Vermeer, M. de Groot, K. V.

Vienola, and J. F. de Boer, Biomed Opt Express 5, 27362758 (2014).

[6] M. Villiger, D. Lorenser, R. A. McLaughlin, B. C. Quirk, R. W. Kirk, B. E. Bouma, and D. D. Sampson, Sci Rep-Uk 6 (2016).

[7] J. Willemse, M.G.O. Gräfe, J. A. van de Kreeke, F. Feroldi, F.D. Verbraak, and J. F. de Boer,). Optics letters, 44(15), 3893-3896 (2019). 